Supporting Materials to

\title{
Rhodium-Catalyzed Cycloisomerization of 1,6-Enynes with an Intramolecular Halogen Shift: Reaction Scope and Mechanism
}

\author{
Xiaofeng Tong, ${ }^{a}$ Dao Li, ${ }^{a}$ Zhaoguo Zhang ${ }^{* a}$ and Xumu Zhang ${ }^{b}$
}

State Key Laboratory of Organometallic Chemistry, Shanghai Institute of Organic Chemistry, Chinese Academy of Science, 354 Fenglin Rd., Shanghai 200032, China and Department of Chemistry, the Pennsylvania State University, University Park, PA 16801, USA

General.

Materials . $\mathrm{S} 2$

General procedure for the cycloisomerization and data for P1-22. S3-S20

Precedure for the synthesis of S11 and S12. $\mathrm{S} 21-\mathrm{S} 23$

GC traces of reaction process for Eq. 5 S24 
General: All reactions and manipulations were conducted in an Argon-filled glovebox or using standard Schlenk techniques. Column chromatography was performed using Silica gel 60 (300-400 mesh). ${ }^{1} \mathrm{H}$ and ${ }^{13} \mathrm{C}$ were recorded on a $300 \mathrm{MHz}$ spectrometers. Chemical shifts were reported in ppm down field from tetramethylsilane with the solvent resonance as the internal standard. MS, IR and microanalysis were done by the authorized analytical center in Shanghai Institute of Organic Chemistry.

Materials: 1,2-dichloroethane was distilled from $\mathrm{P}_{2} \mathrm{O}_{5}$ prior to use. The silver salt $\mathrm{AgSbF}_{6}$ and $\mathrm{RhCl}_{3}$ were purchased from Aldrich. $[\mathrm{Rh}(\mathrm{COD}) \mathrm{Cl}]_{2},{ }^{1}\left[\mathrm{Rh}(\mathrm{COD})_{2}\right] \mathrm{SbF}_{6},{ }^{2} \mathrm{Rh}(\mathrm{CO}) \mathrm{Cl}\left(\mathrm{PPh}_{3}\right)_{2},{ }^{3}$ $[\mathrm{Rh}(\mathrm{dppb}) \mathrm{Cl}]_{2}{ }^{4}$ and $\mathrm{RhCl}\left(\mathrm{PPh}_{3}\right)_{3}{ }^{5}$ were prepared according to the reported literature procedures.

${ }^{1}$ Giordano, G.; Crabtree, R. H. Inorg. Synth. 1990, 28, 88.

${ }^{2}$ Schenck, T. G.; Downes, J. M.; Milne, C. R. C.; Mackenzie, P. B.; Boucher, H.; Whelan, J.; Bosnich, B. Inorg. Chem. 1985, 24, 2334.

${ }^{3}$ Evans, D.; Wilkinson, G. Inorg. Synth. 1990, 28, 79.

${ }^{4}$ Fairlie, D. P.; Bosnich, B. Organometallics 1998, 7, 936.

${ }^{5}$ Osborn, J. A.; Wilkinson, G. Inorg. Synth. 1990, 28, 77 
General procedure for cycloisomerization of enyne with $\operatorname{RhCl}\left(\mathrm{PPh}_{3}\right)_{3}$ : Under $\operatorname{Argon}$ atmosphere, a $25 \mathrm{ml} \mathrm{Schlenk} \mathrm{tube} \mathrm{is} \mathrm{charged} \mathrm{with} 0.2 \mathrm{mmol}$ of enyne substrate, $10 \mathrm{~mol} \%$ of $\mathrm{RhCl}\left(\mathrm{PPh}_{3}\right)_{3}$ and $3 \mathrm{~mL}$ of $\mathrm{ClCH}_{2} \mathrm{CH}_{2} \mathrm{Cl}$ (DCE). The mixture was stirred under reflux. The reaction was monitored by TLC. After the reaction was complete, the reaction mixture is directly subjected to column chromatography using PE-EtOAc (20:1-10:1) as eluent.

\section{P1 (Table 2, entry 1):}<smiles>C=CC1COC(=O)C1=C(C)Cl</smiles>
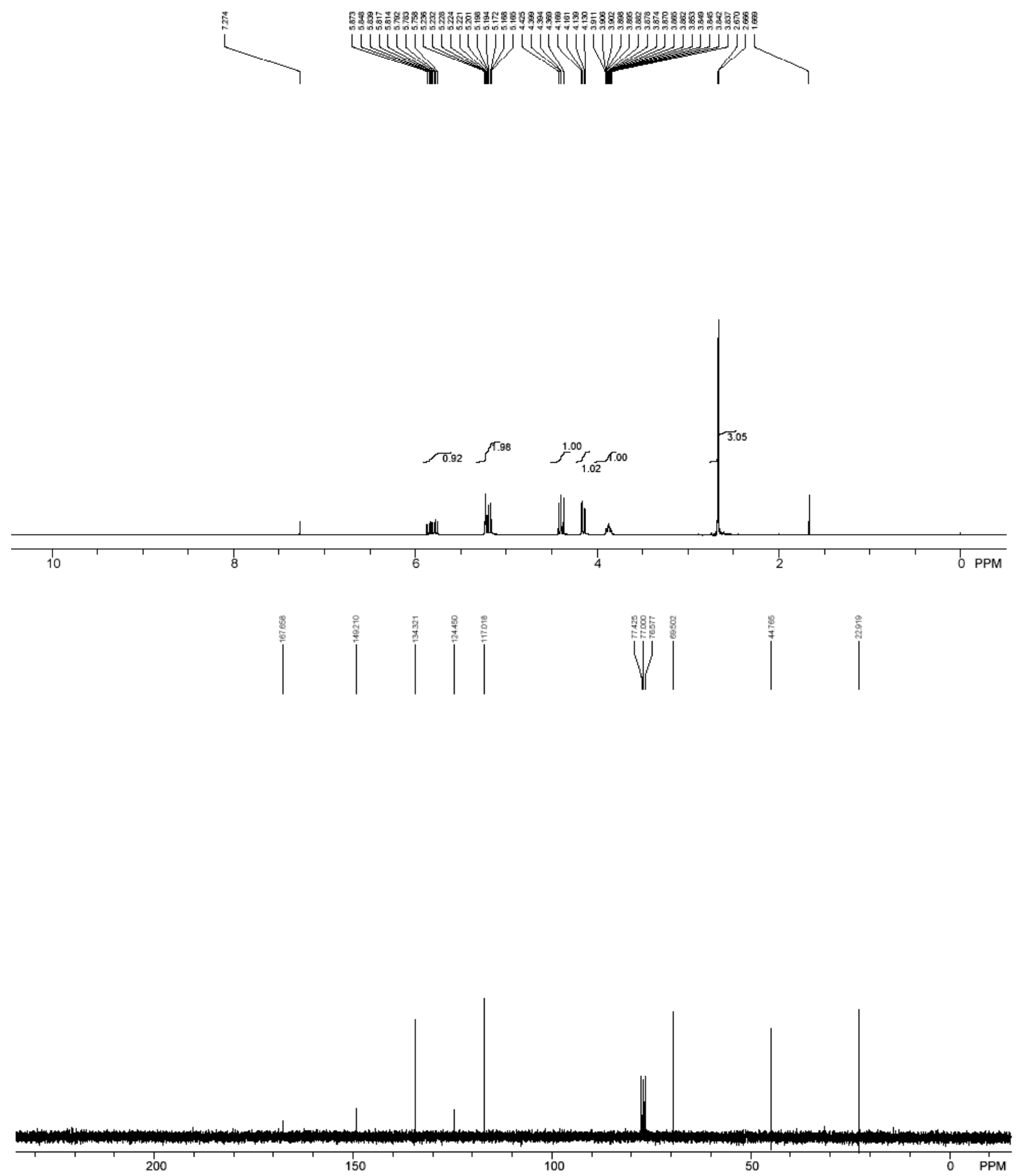
P2 (Table 2, entry 2):<smiles>C=CC1COC(=O)C1=C(Cl)c1ccccc1</smiles>

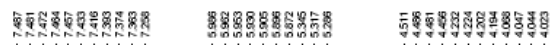

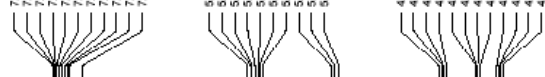

$$
\text { \̊ }
$$
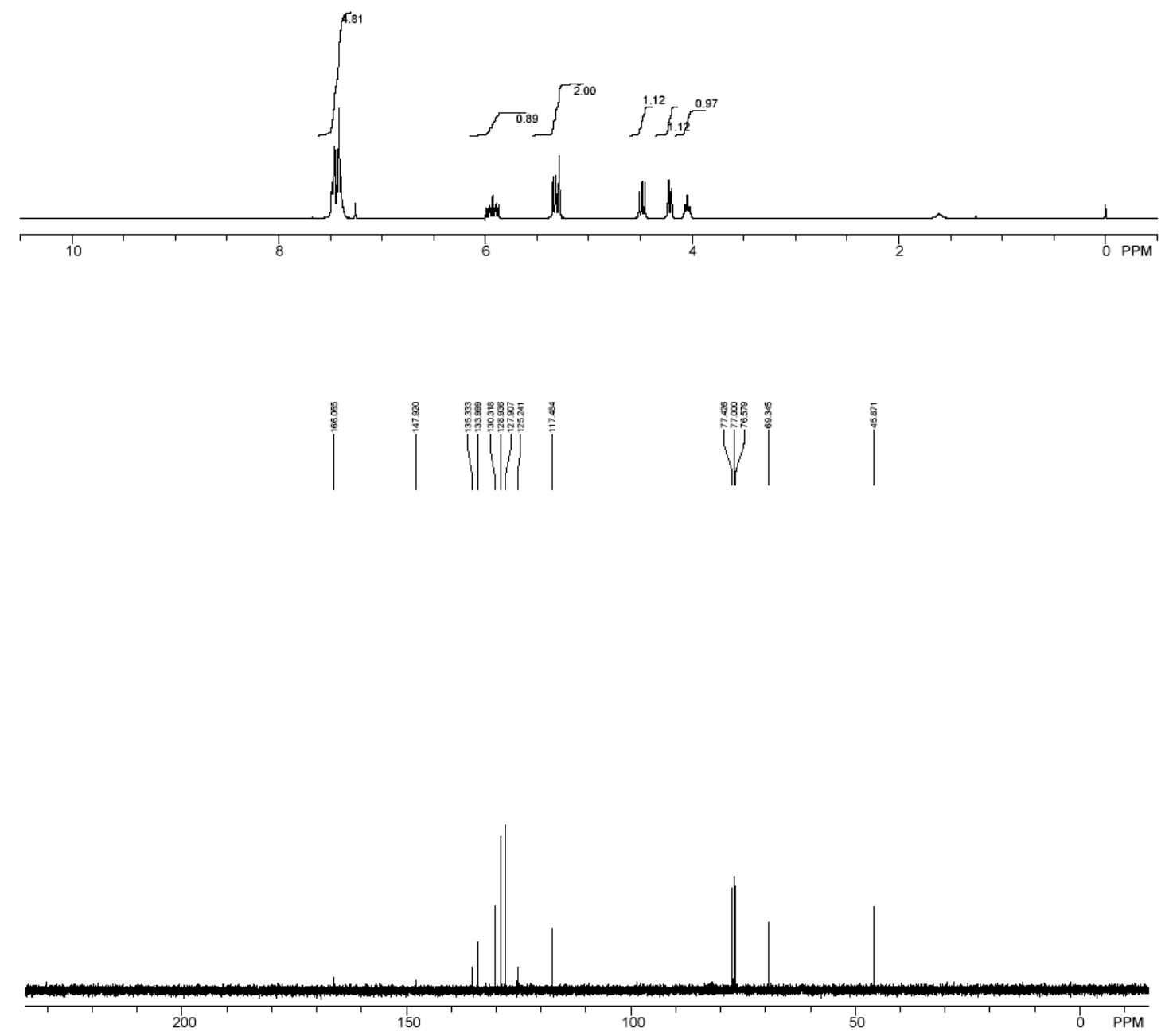
P3 (Table 2, entry 3):<smiles>C=CC1COC(=O)C1=C(Cl)Br</smiles>

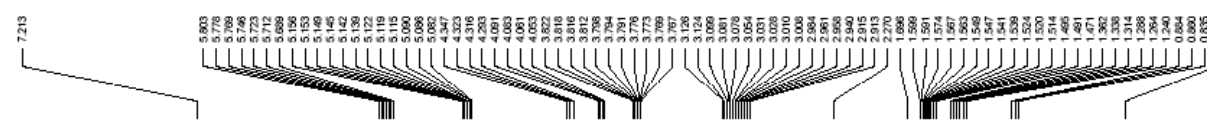
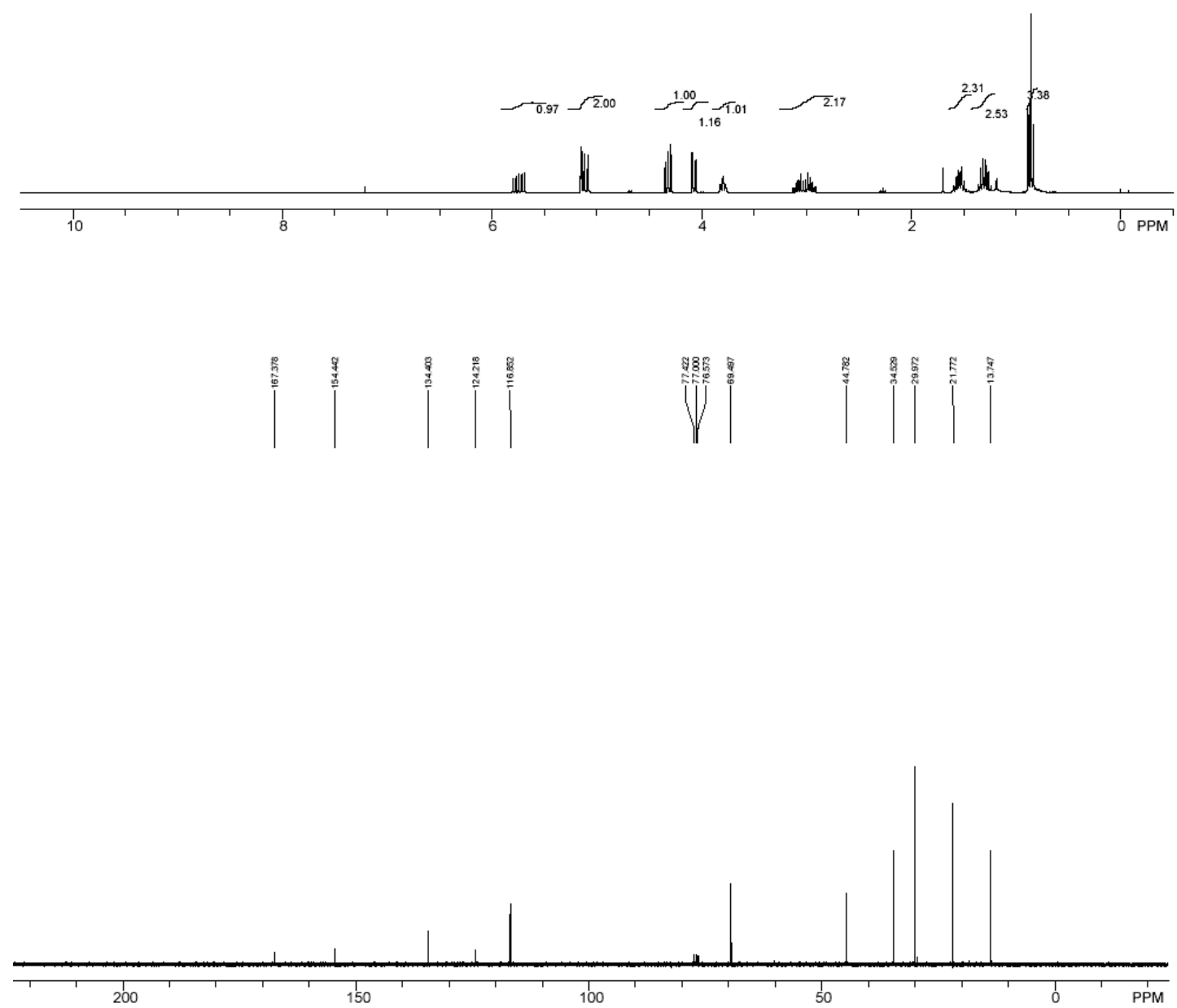
P4 (Table 2, entry 4):<smiles>C=CC1COC(=O)C1=C(Cl)COC</smiles>

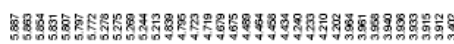

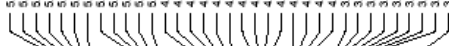

Y W W
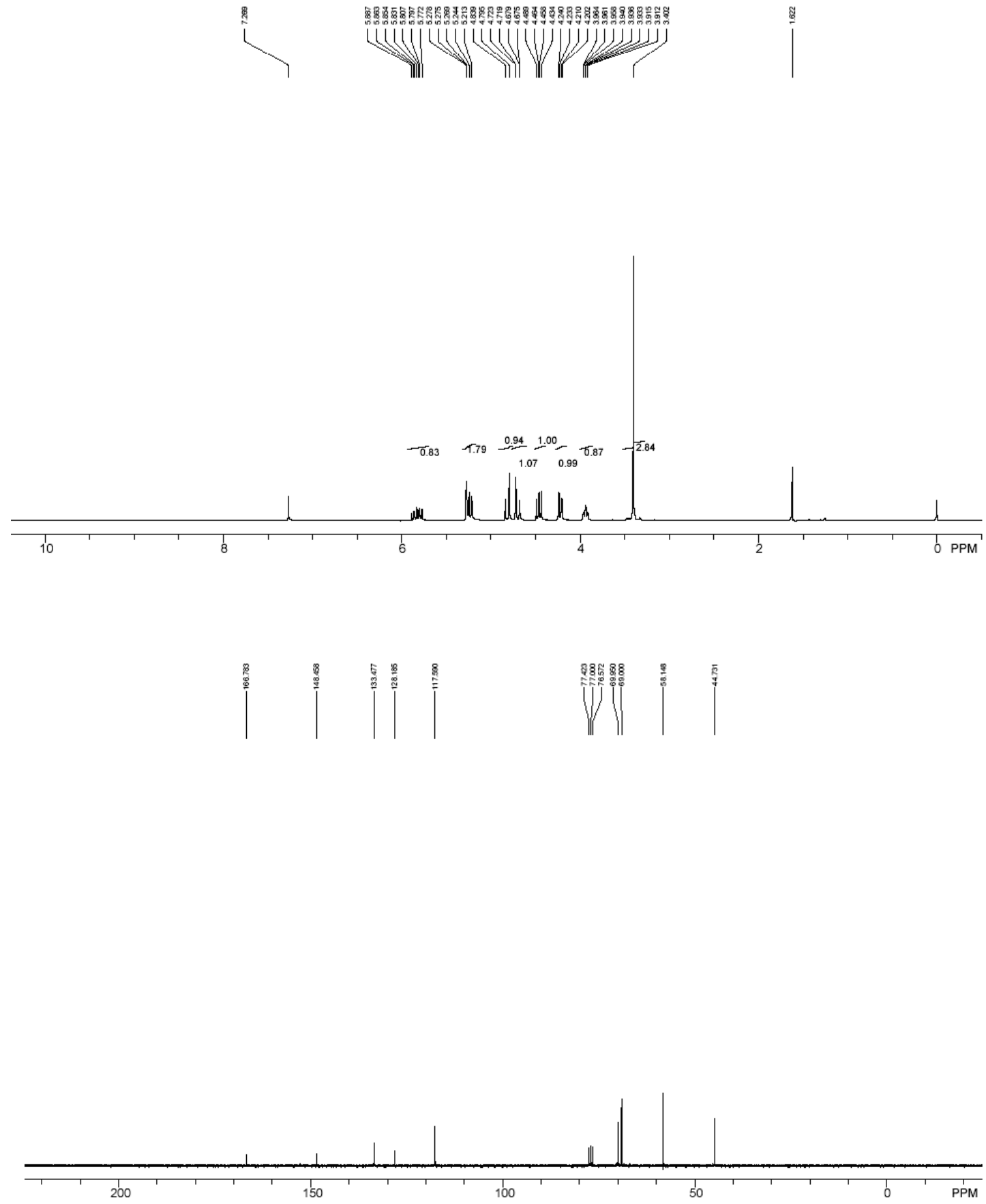
$\mathrm{P5}^{6}$ (Table 2, entry 5):<smiles>C=CC1COC(=O)/C1=C/Cl</smiles>
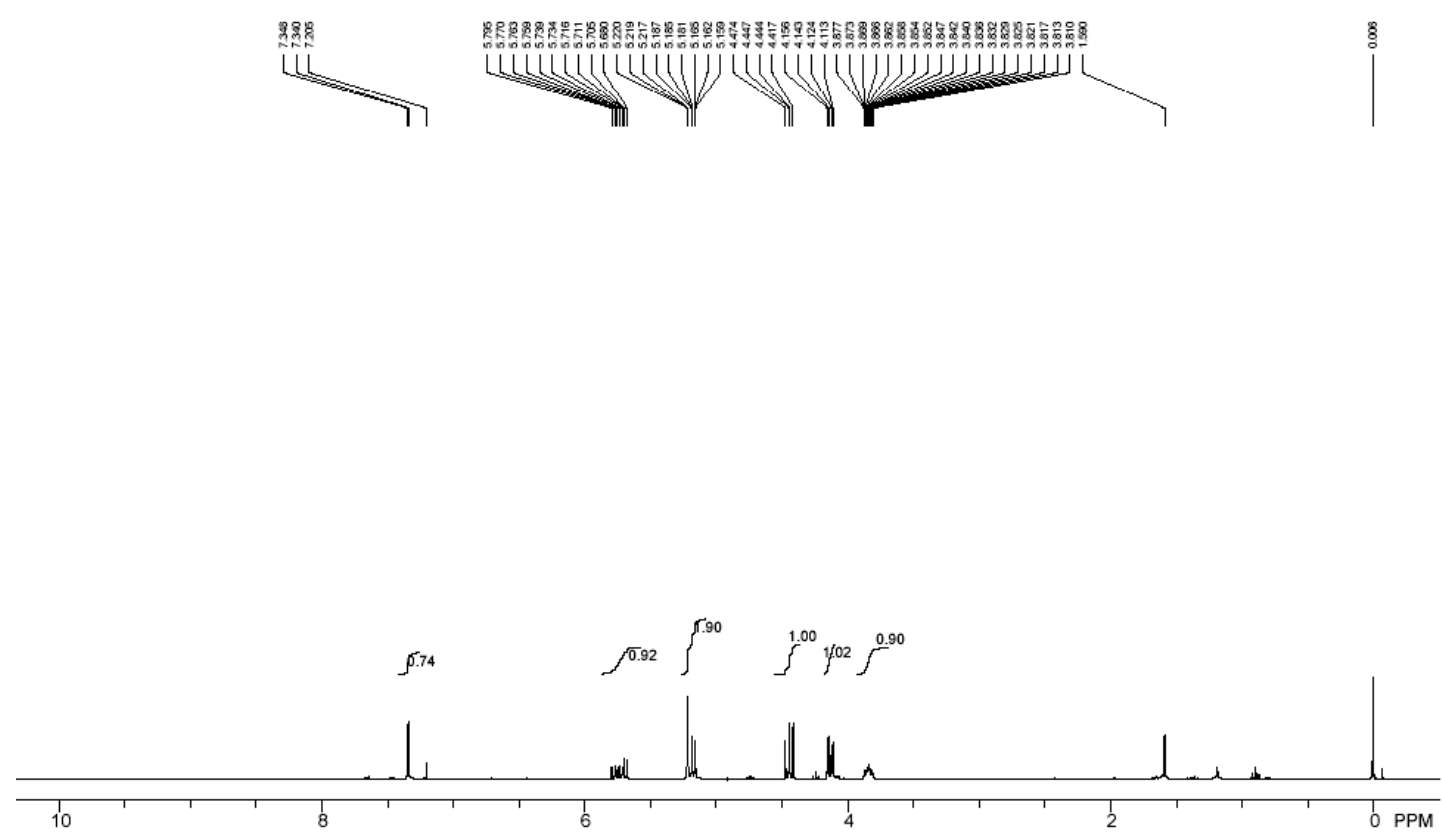

6 (a) Ma, S.; Lu, X. J. Org. Chem. 1991, 56, 5120. (b) Zhu, G.; Ma, S.; Lu, X. J. Chem. Res. Miniprint 1993, 9 , 2467. 
P6 (Table 3, entry 7):

$\mathrm{BnOCH}_{2}$

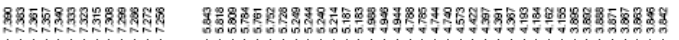

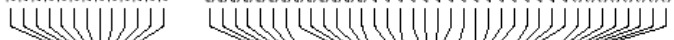

(III

+ II
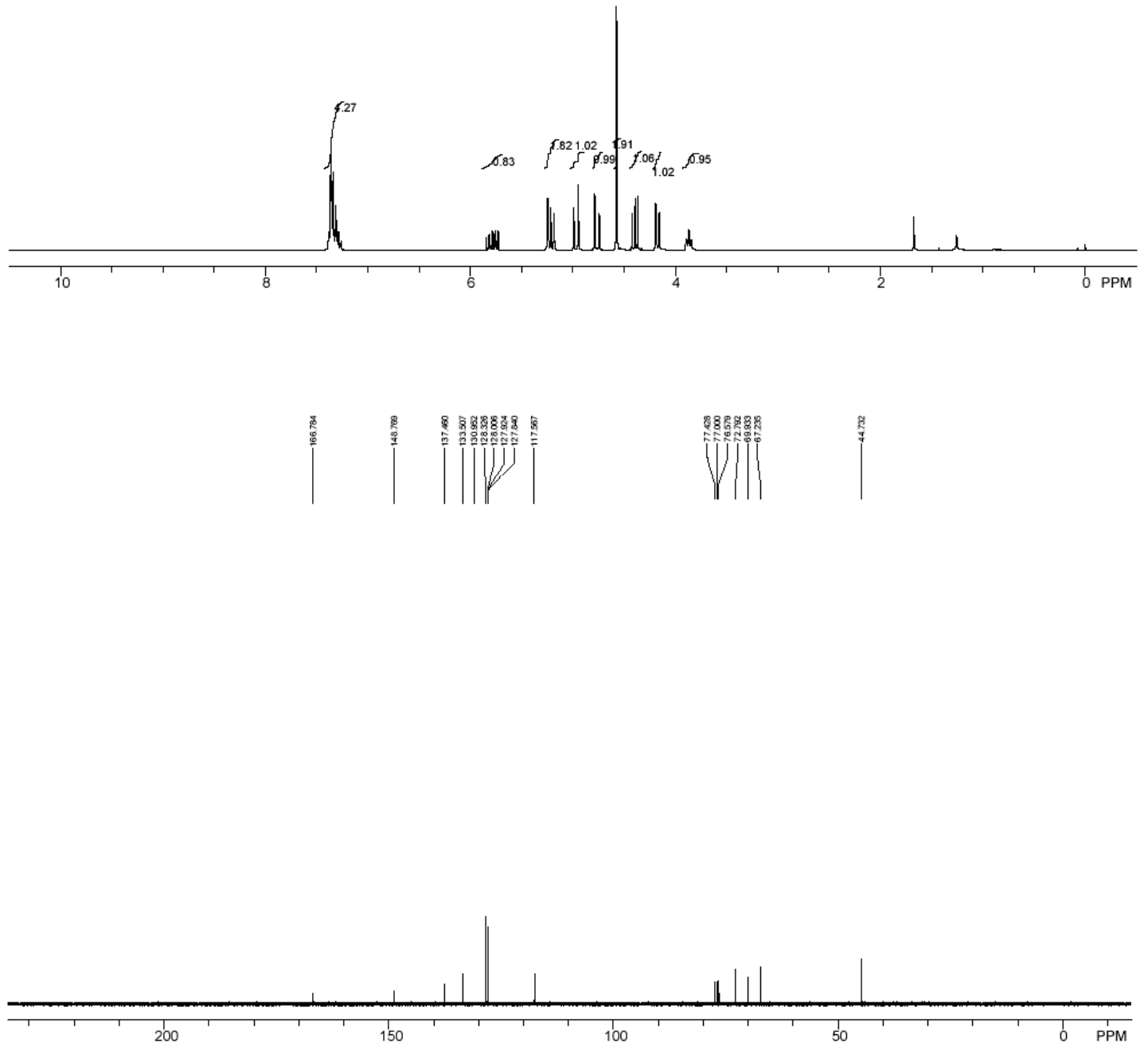
P7 (Table 3, entry 8):<smiles>C=CC1CNC(=O)C1=C(C)Cl</smiles>

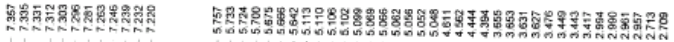

txf-4-58

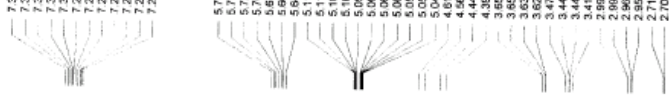

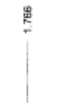
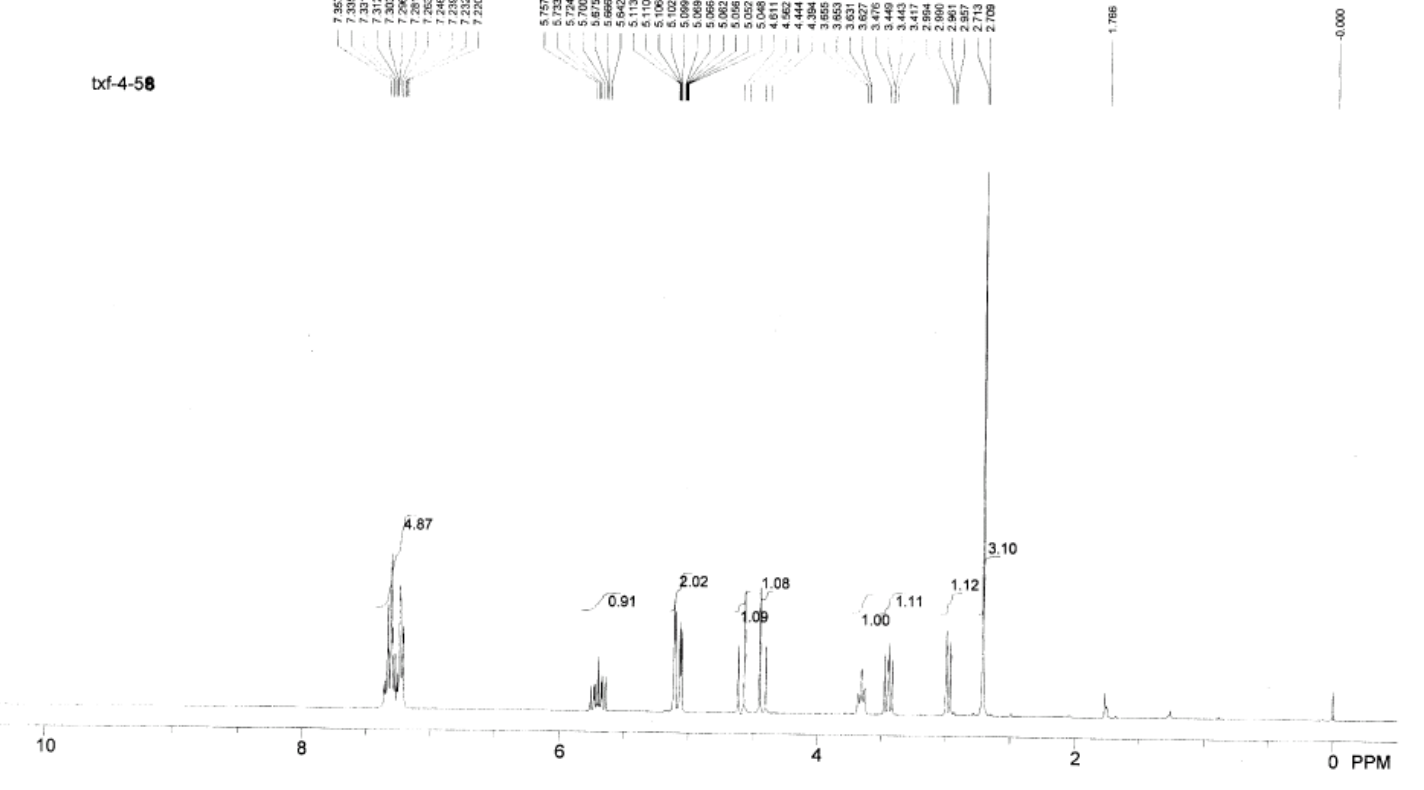

Archive directory: /export/home/1u-xy/vnarsys/aata
Sample directory:
File: CARBoN

Pulse Sequence: s2pur

Solvent: : CDC13
Amb ent temperature
Mercury-300BB
Menc300"

Relax. de lay 1.000 sec

Pulse 45.0 degrees

584 repetitions
OBSERVE C13. $75.4421005 \mathrm{MHz}$

0
0

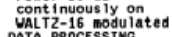

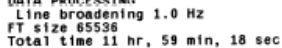
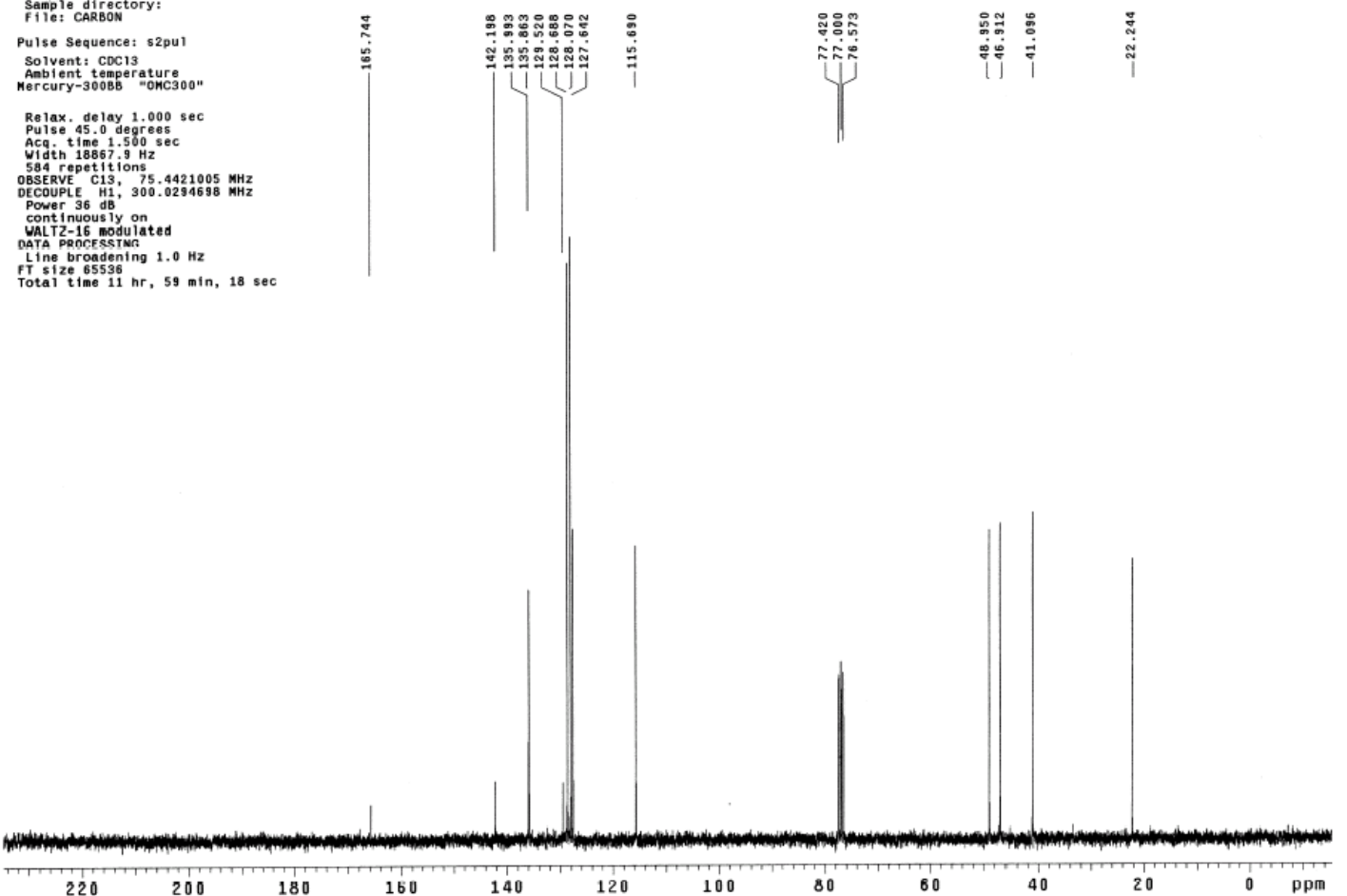
P8 (Table 3, entry 9):<smiles>C=CC1CN(Cc2ccccc2)C(=O)/C1=C(/Cl)c1ccccc1</smiles>

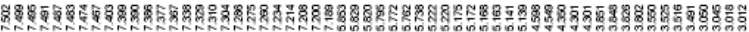

Li(i)Lu(iu)
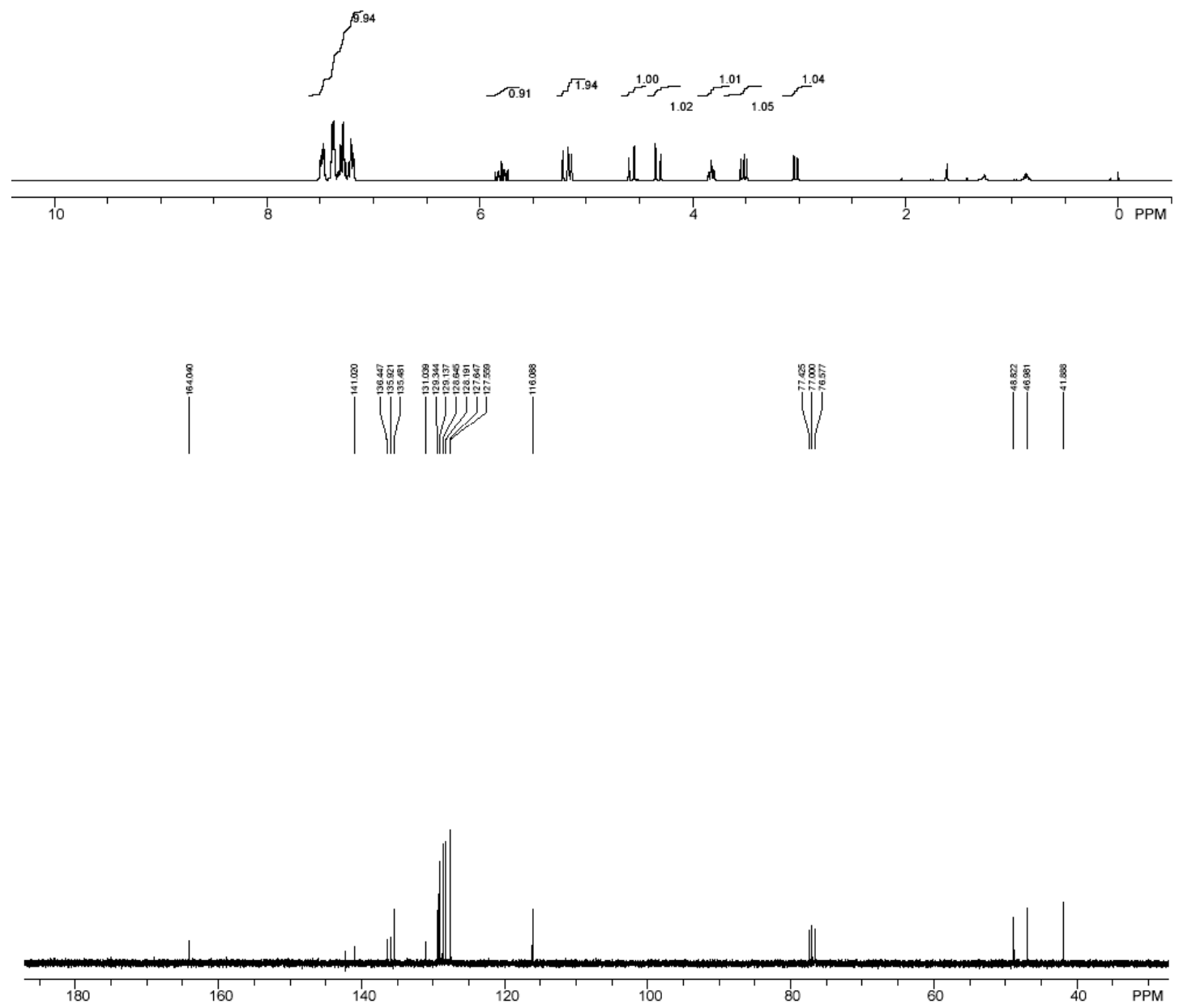
P9 (Table 3, entry 10):<smiles>C=CC1CN([As])C(=O)C1=C(C)Cl</smiles><smiles>[V]=CC=[Tl]</smiles>
WU
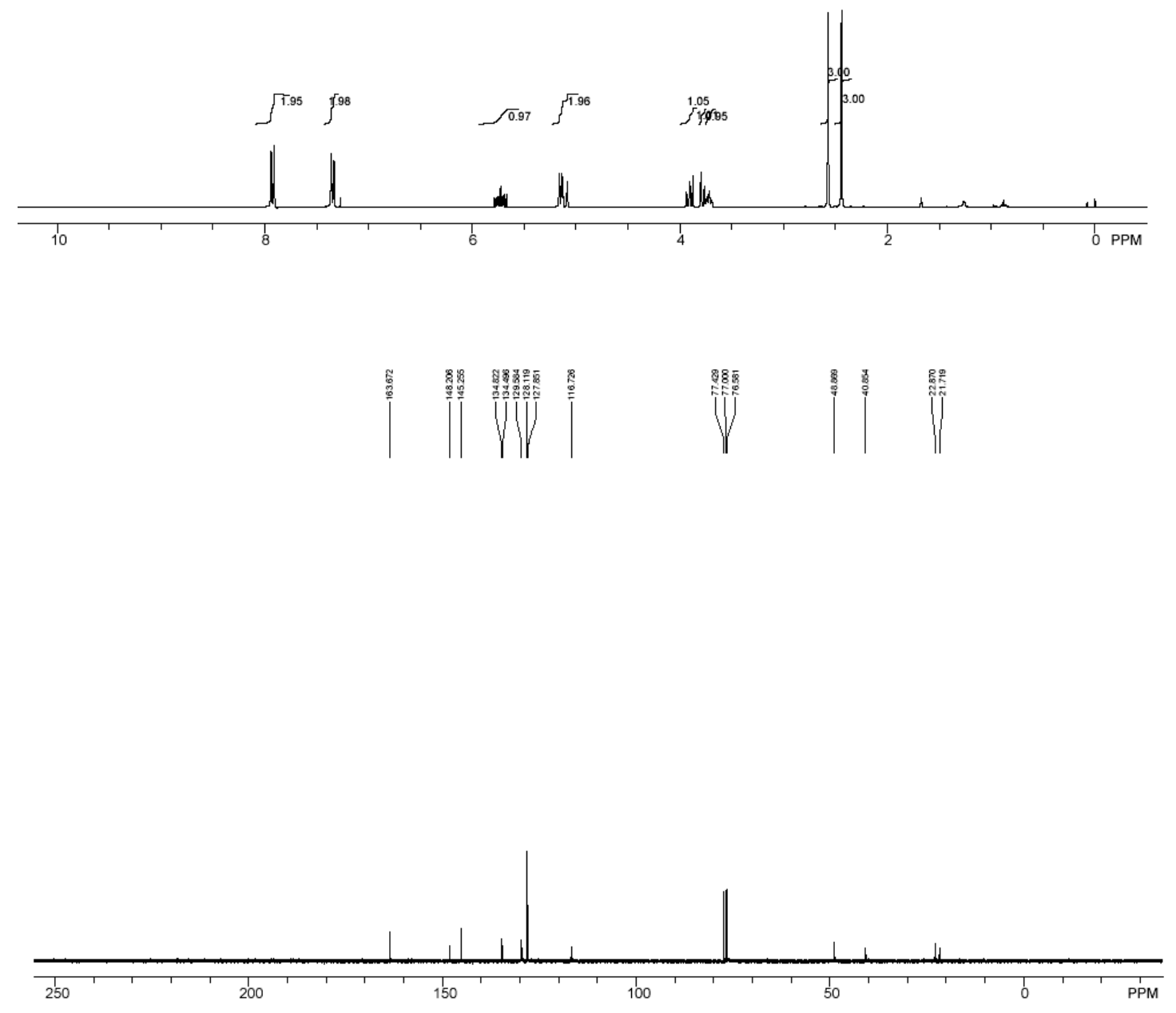
P14:

$=$
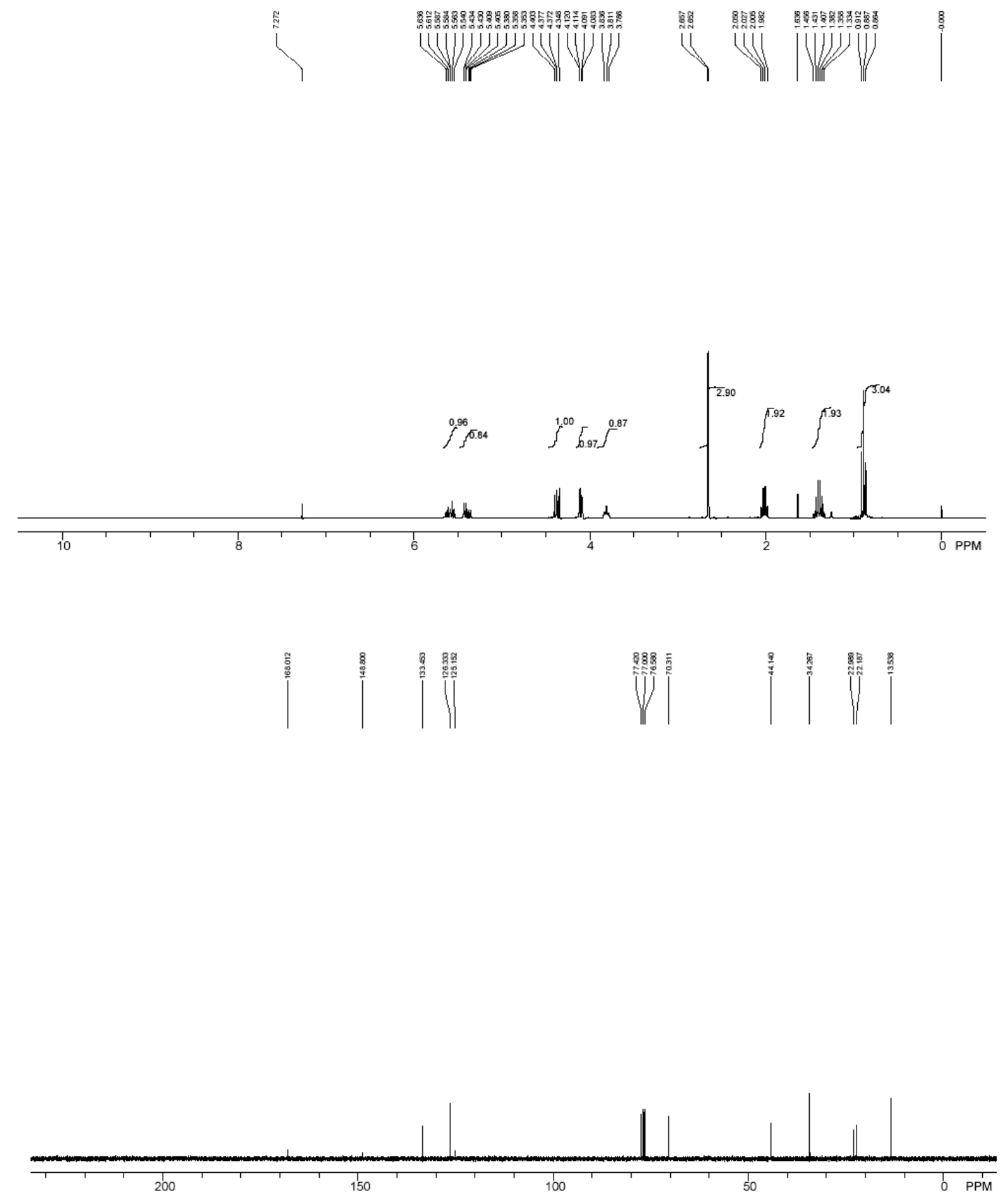
P15:

$\int_{0}$

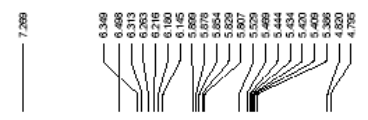

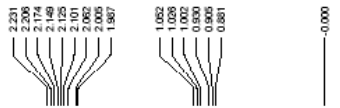

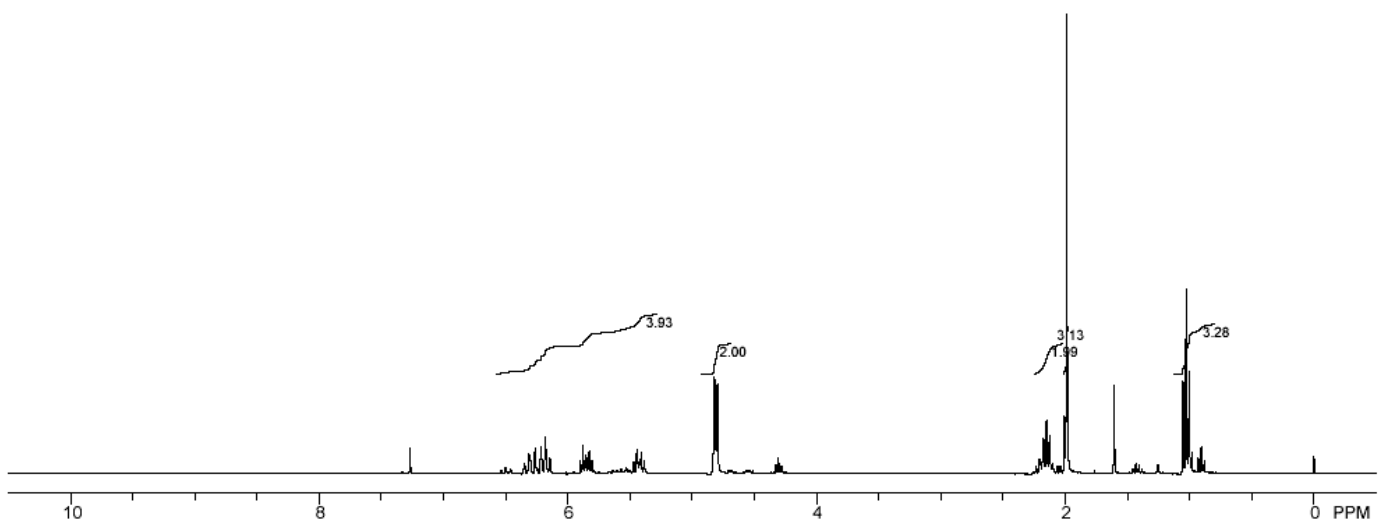


P16 (Table 4, entry 1):<smiles>C=CC1COCC1=C(C)Cl</smiles>

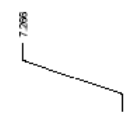

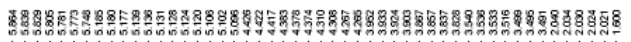

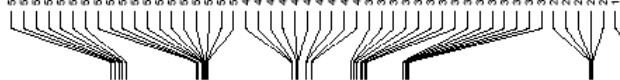
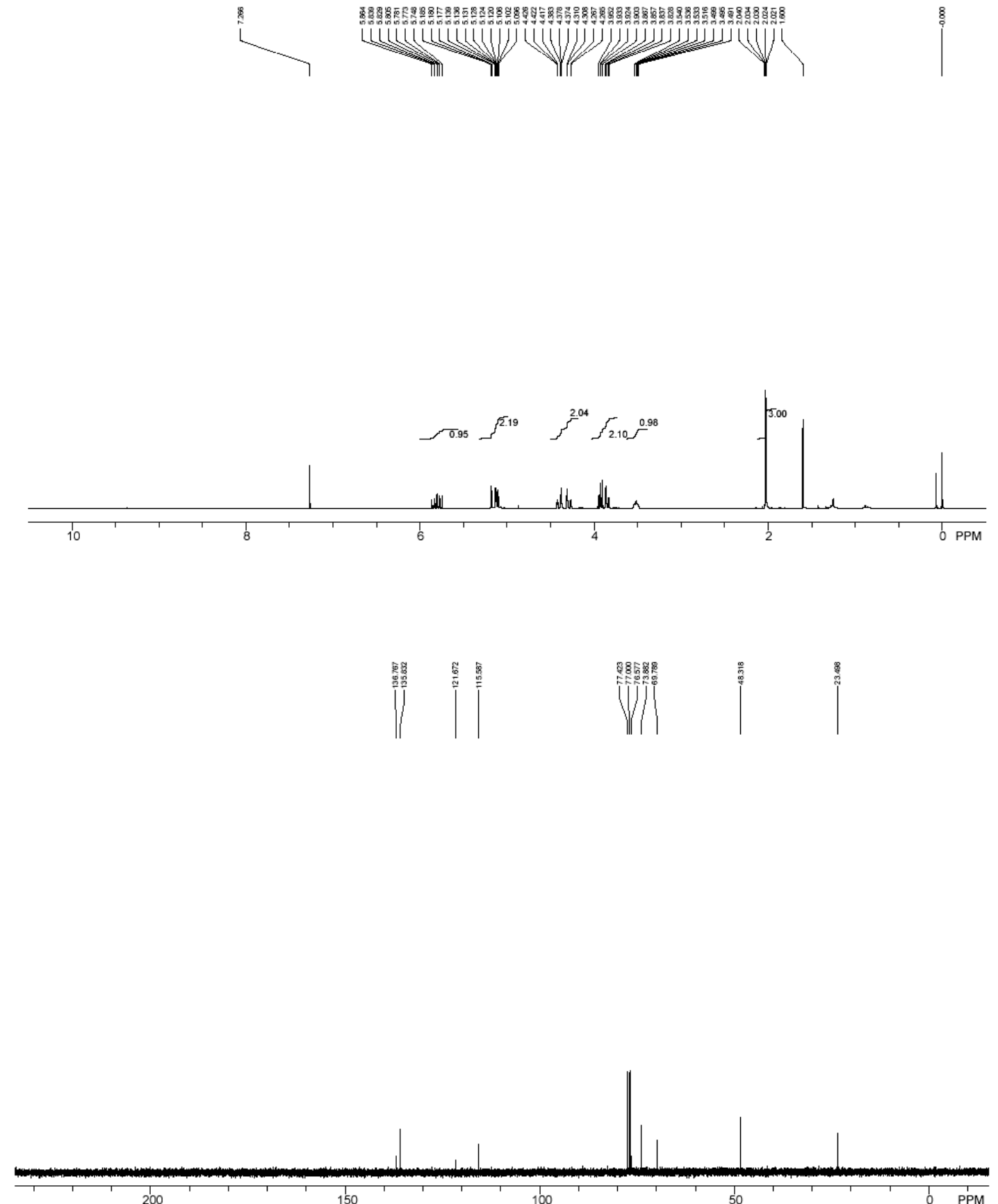
P17 (Table 4, entry 2):

$=$

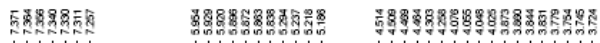

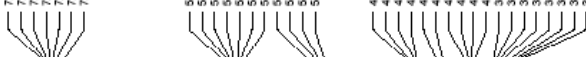
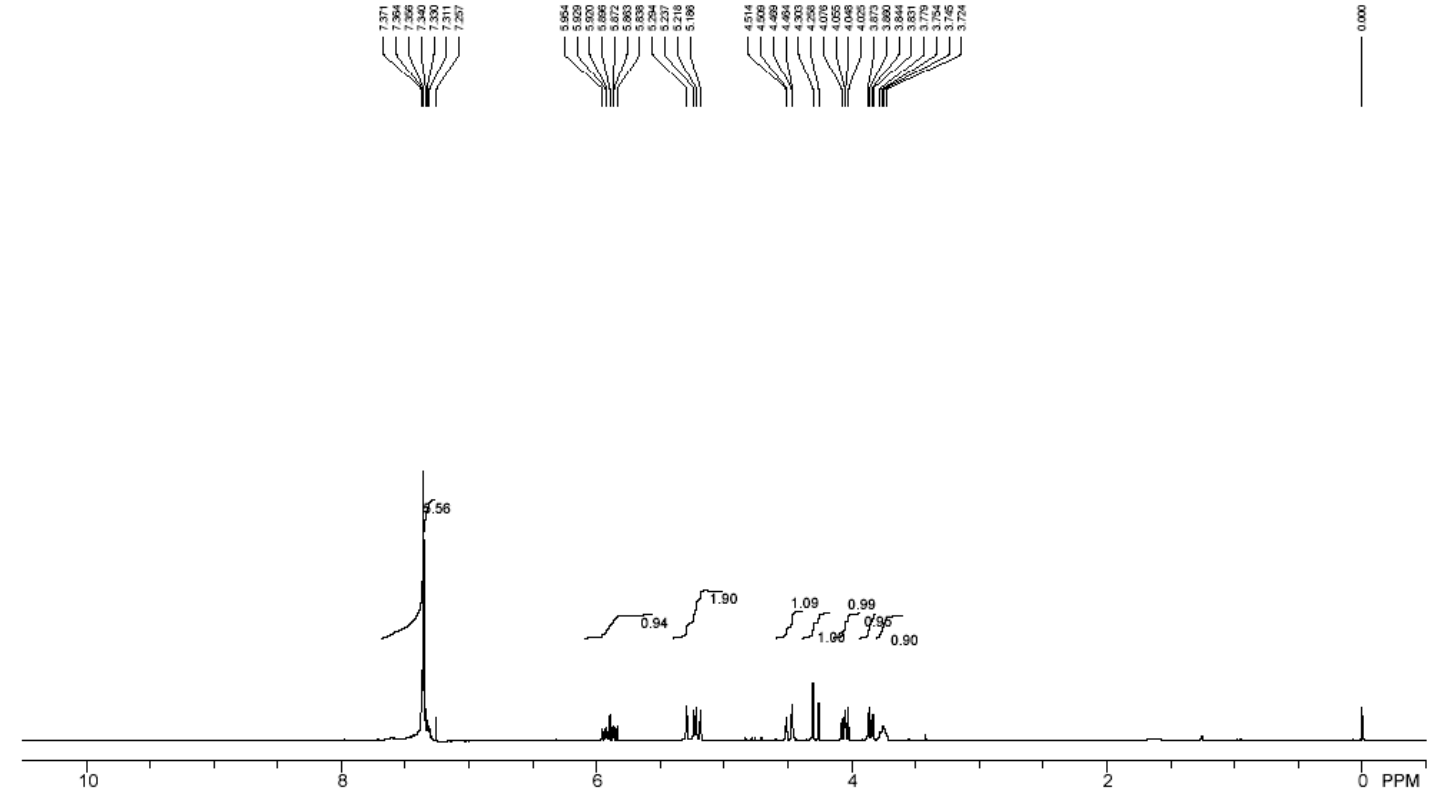

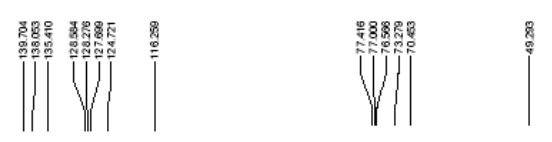

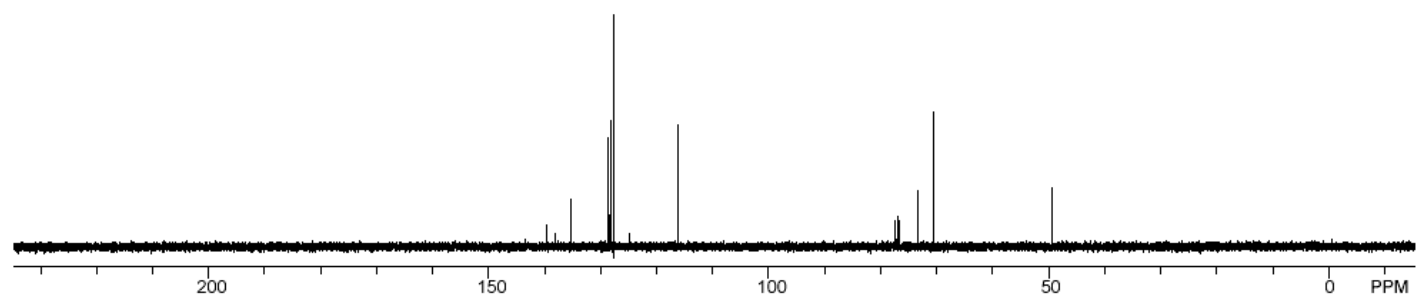


P18 (Table 4, entry 3):

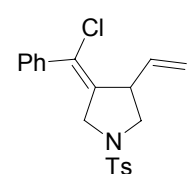

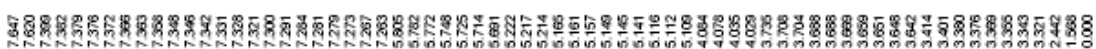

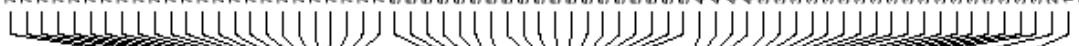

71
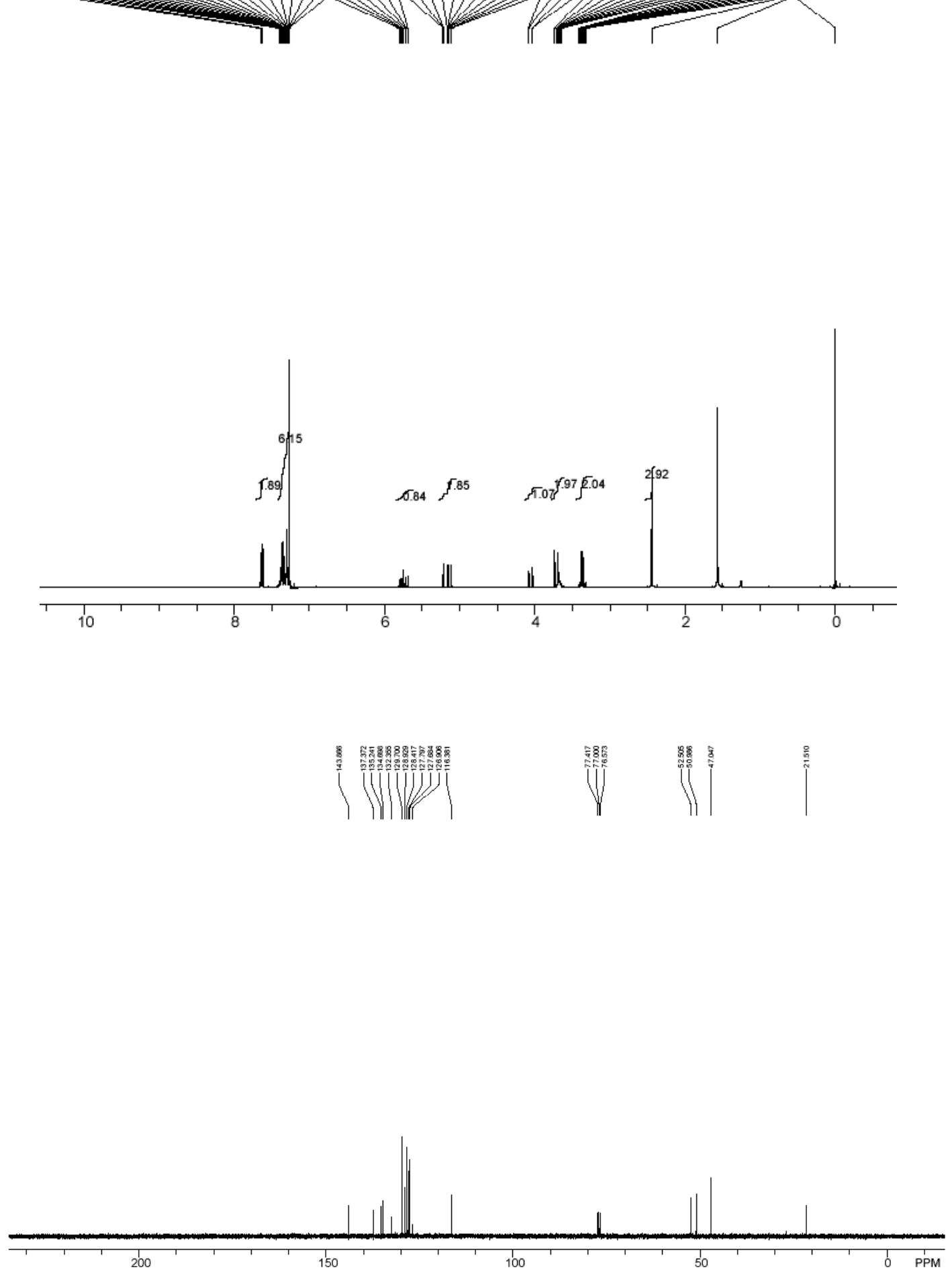
P19 (Table 4, entry 4):<smiles>C=CC1CN(S)CC1=C(Cl)CBr</smiles>

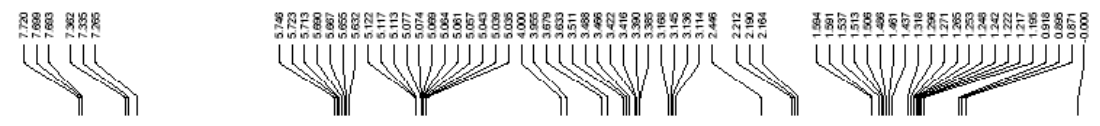
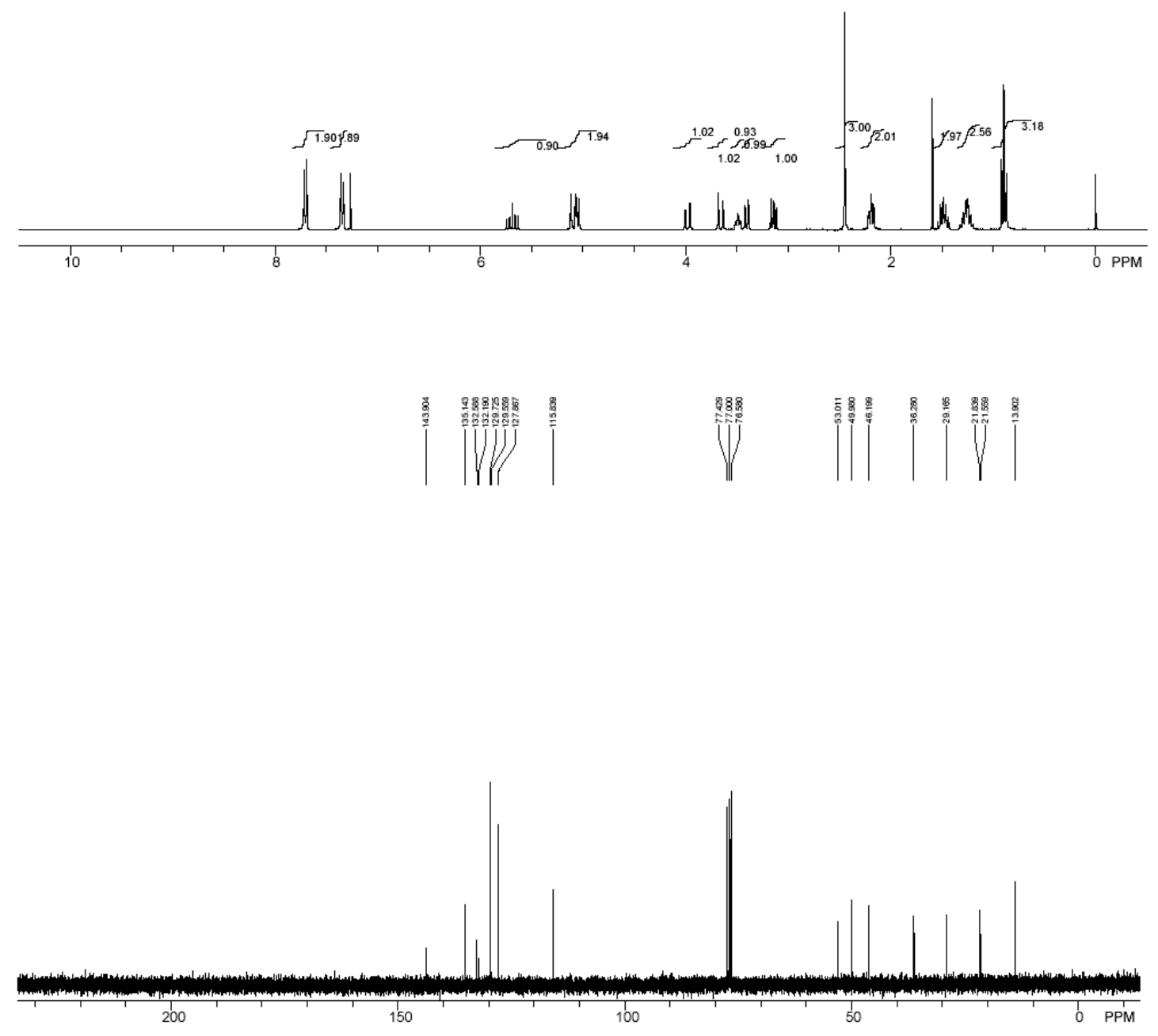
P20 (Table 4, entry 5):
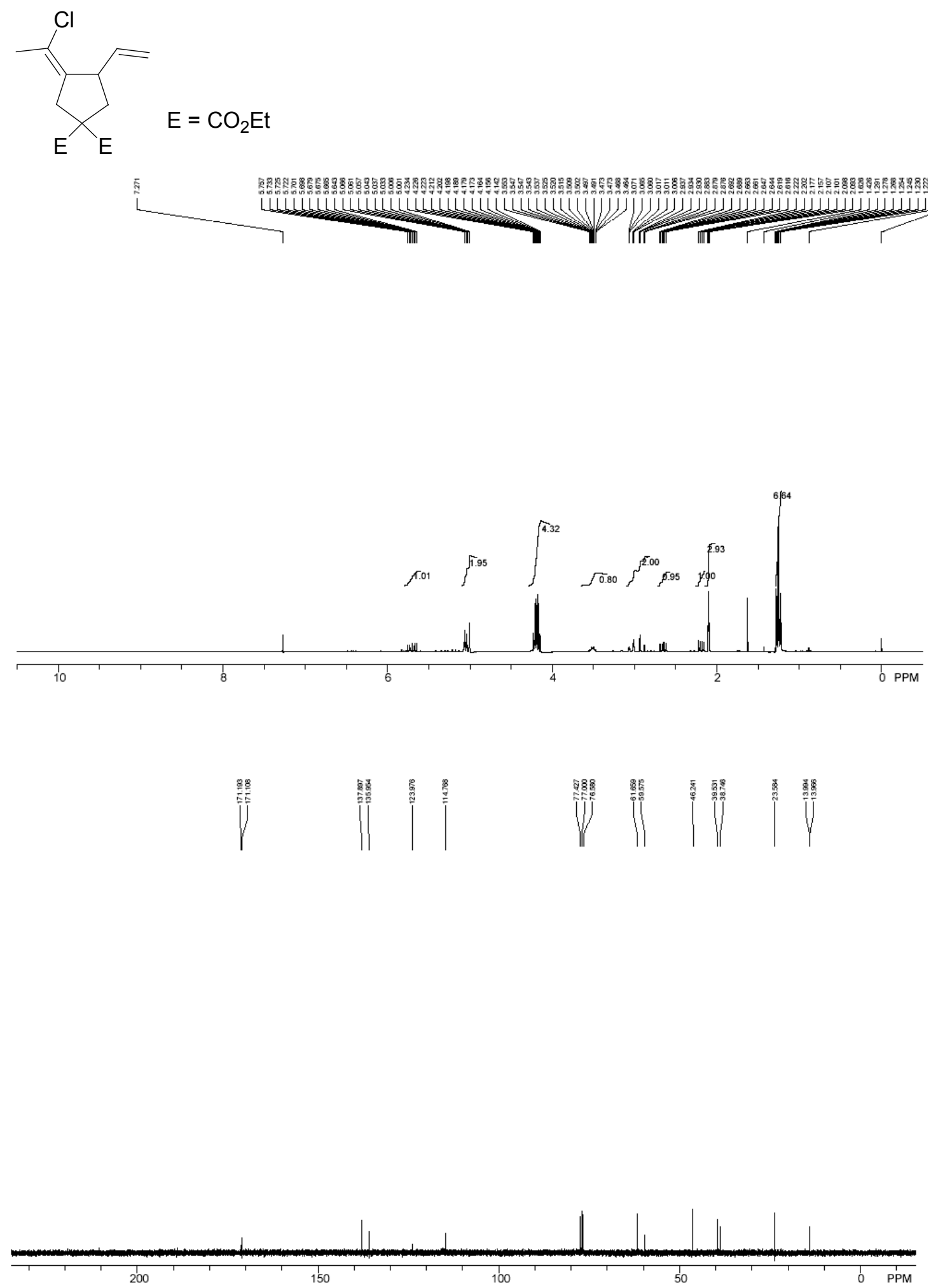
P21 (Table 4, entry 6):

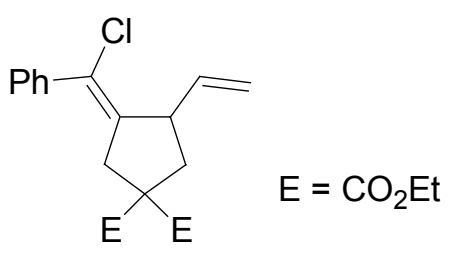

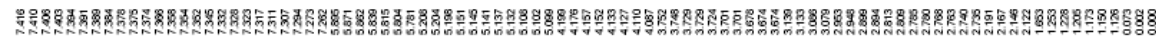

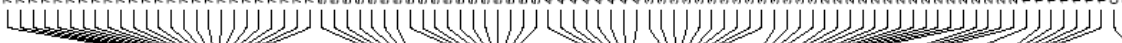
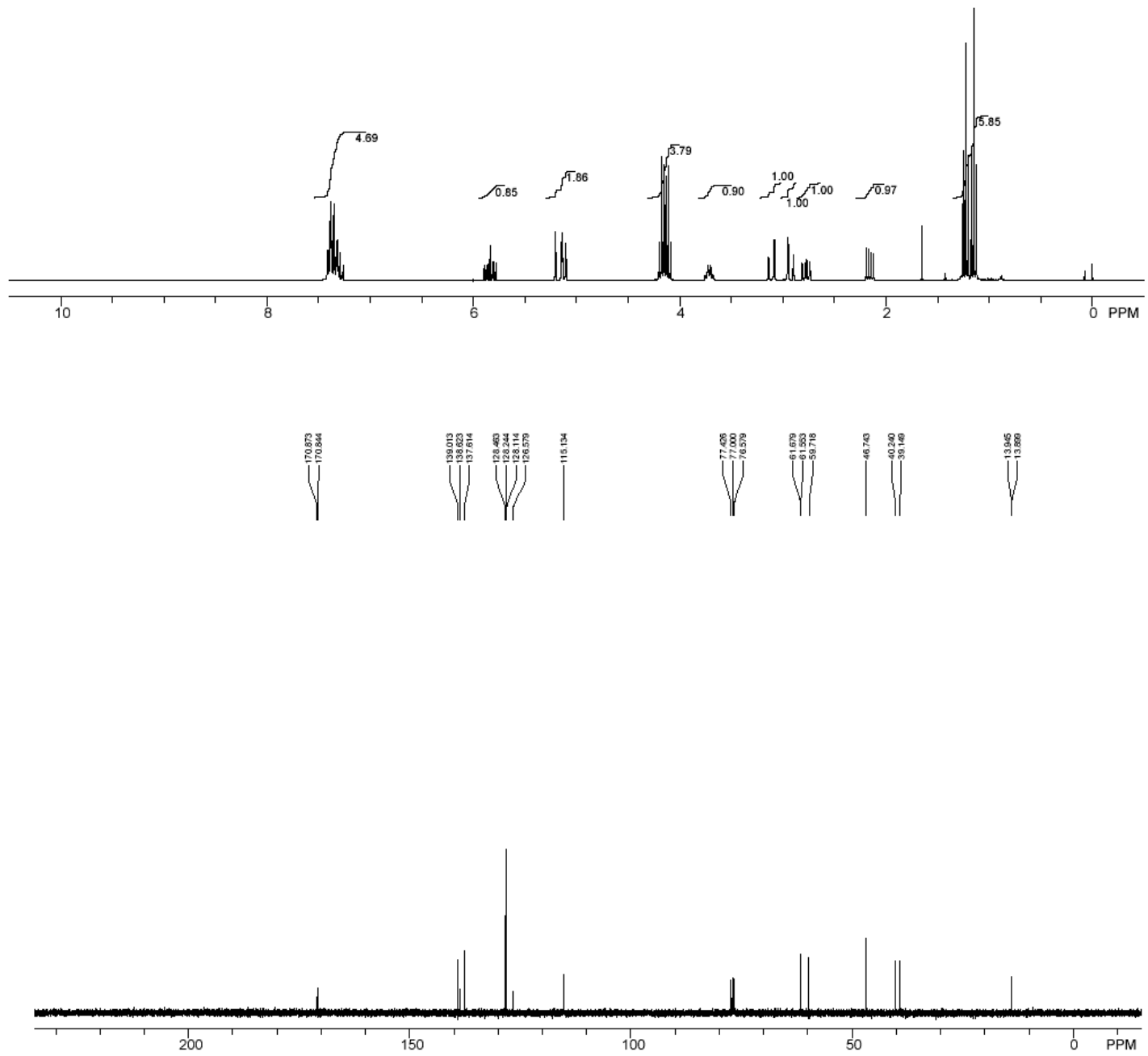
P22 (Table 4, entry 7):
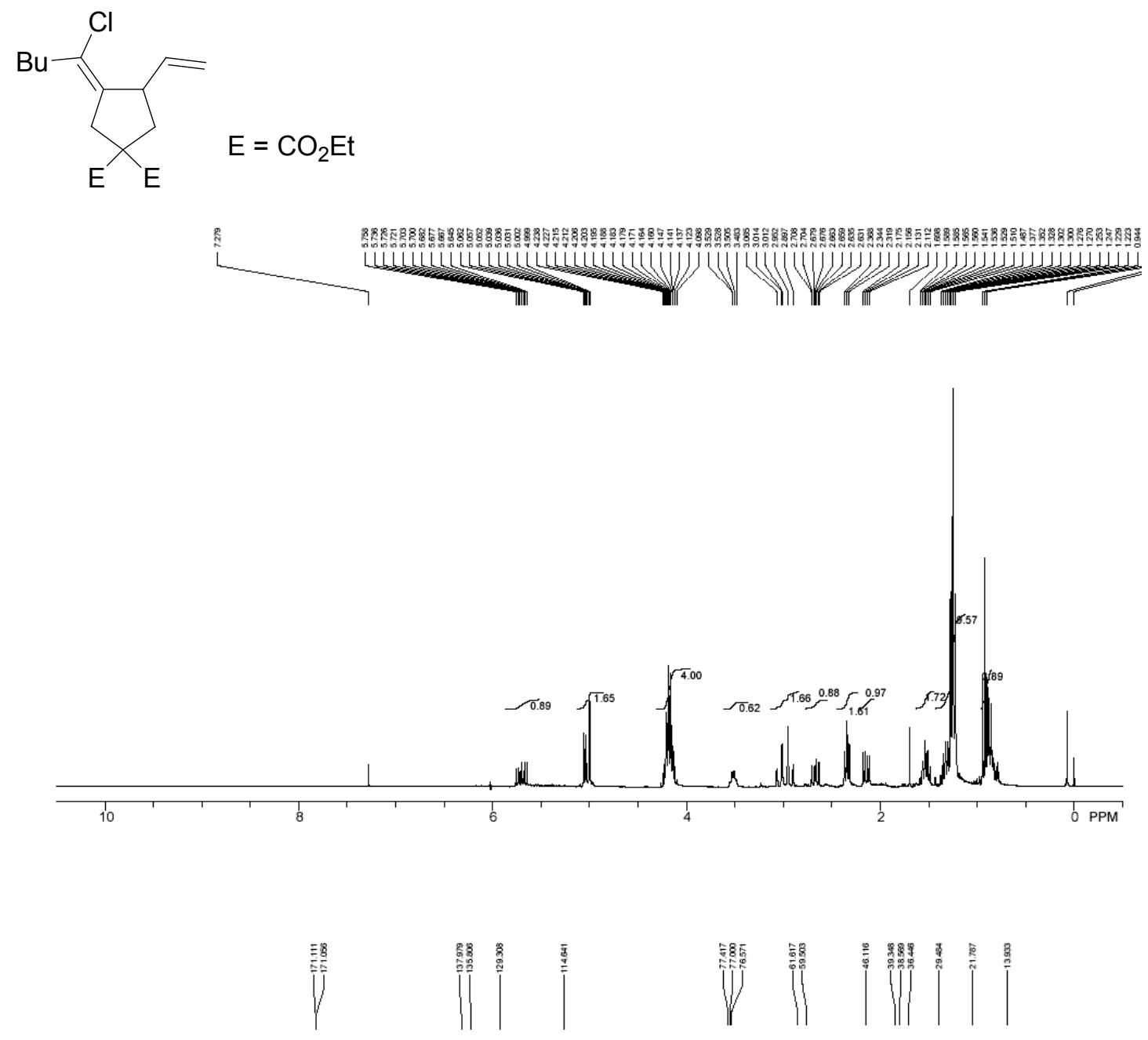
Procedure for the Syntheses of Compound S11 and S12: In a three-necked flask, the substituted propynoic acid (5 mmol) and 2-chloro-3-butene-1-ol $(0.53 \mathrm{~g}, 5 \mathrm{mmol})$ in $\mathrm{CH}_{2} \mathrm{Cl}_{2}(10 \mathrm{~mL})$ was stirred at $-20^{\circ} \mathrm{C}$ under Ar. To this mixture was dropwisely added a solution of DCC (1.03 g, 5 mmol) and DMAP $(6 \mathrm{mg}, 0.05 \mathrm{mmol})$ in $\mathrm{CH}_{2} \mathrm{Cl}_{2}(10 \mathrm{~mL})$. After 6 hours, the reaction mixture was filtrated and the residue was washed by $\mathrm{Et}_{2} \mathrm{O}$. After the solvent evaporating, the residue was subjected to column chromatography to obtain the desired product. 
$\int_{0}$
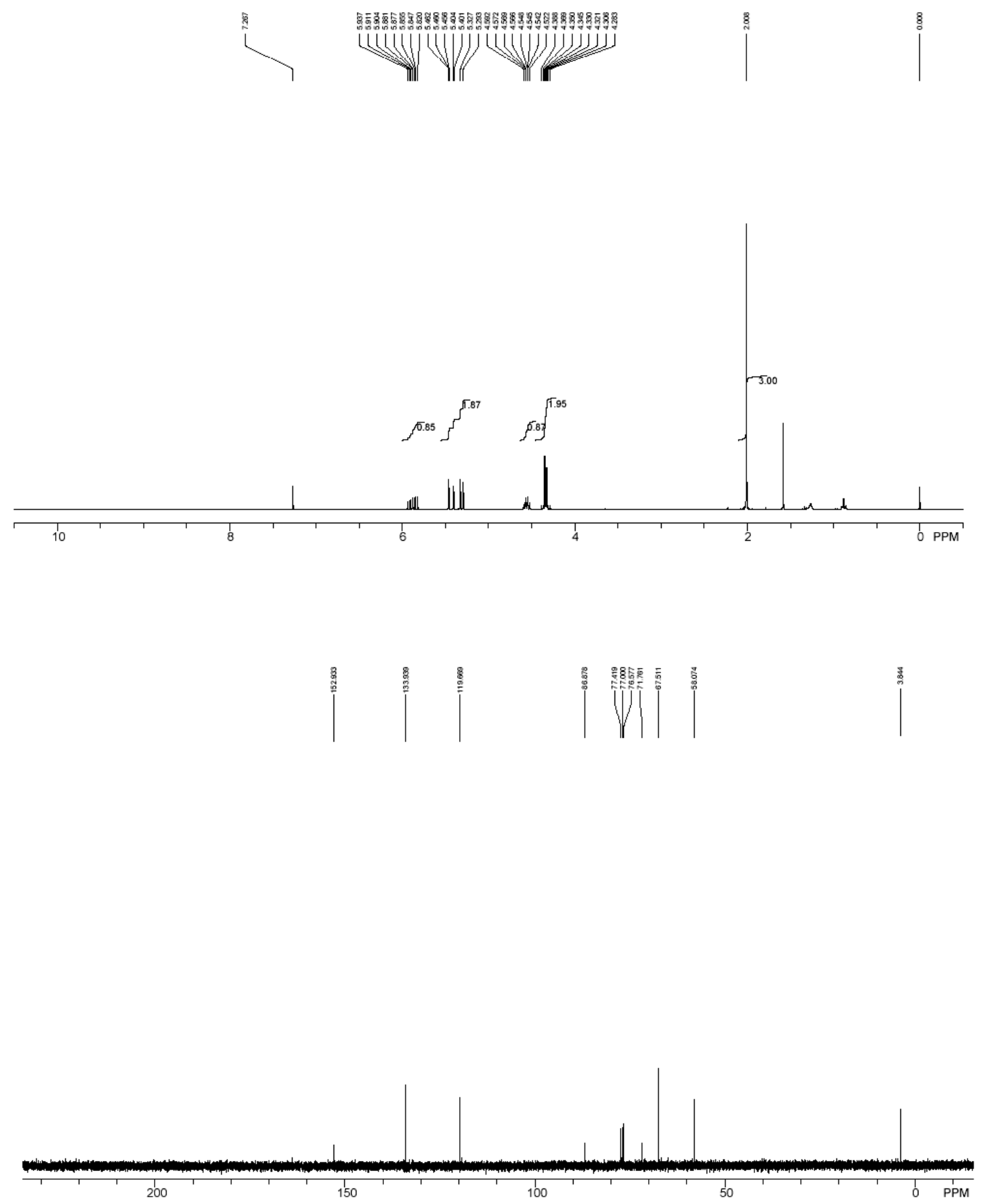


\section{S12}<smiles>C=CC(Cl)COC(=O)C#Cc1ccccc1</smiles>

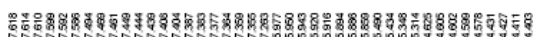

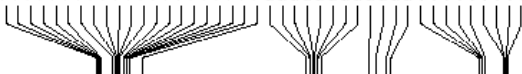
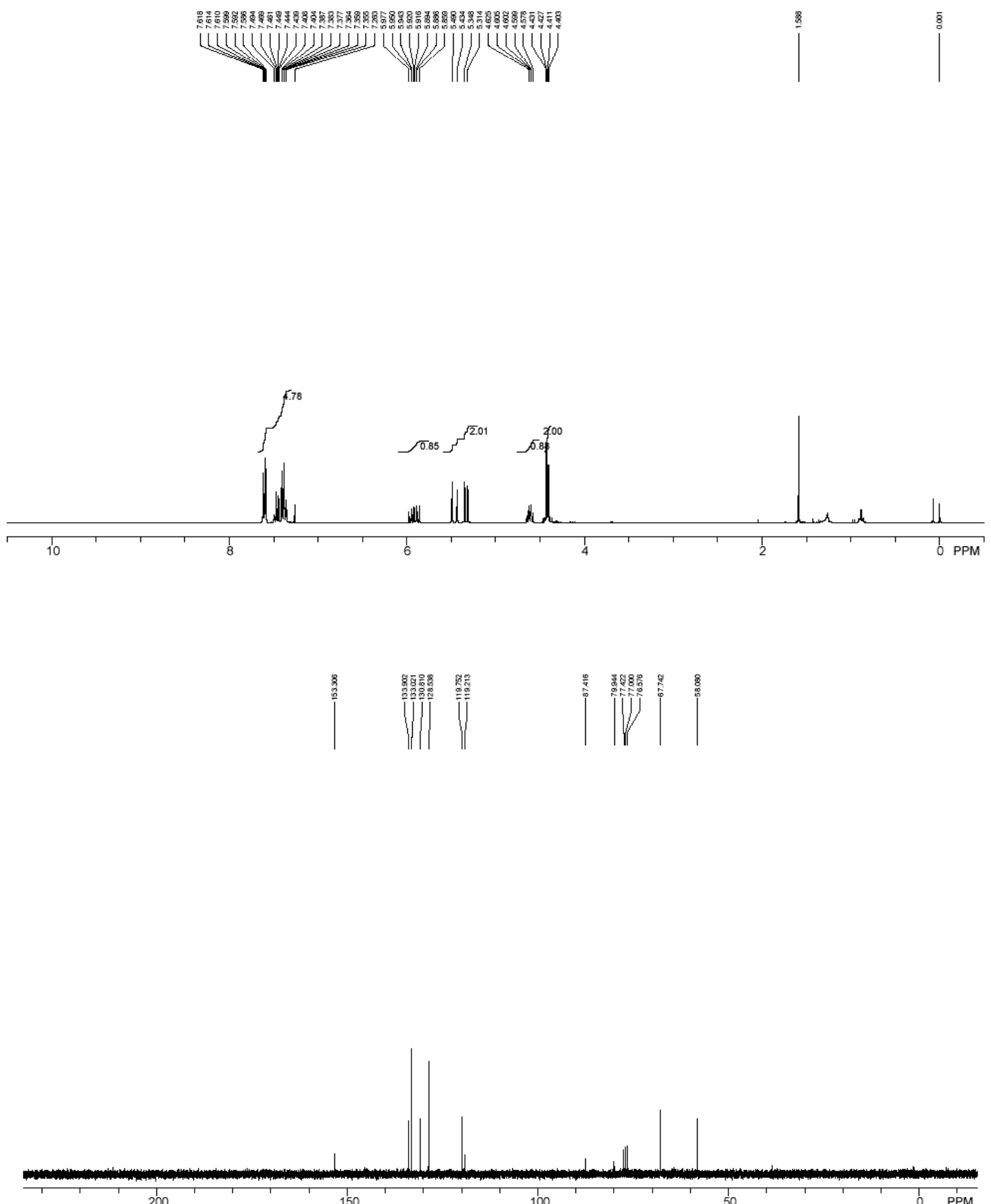
GC traces of reaction process for Eq. 5
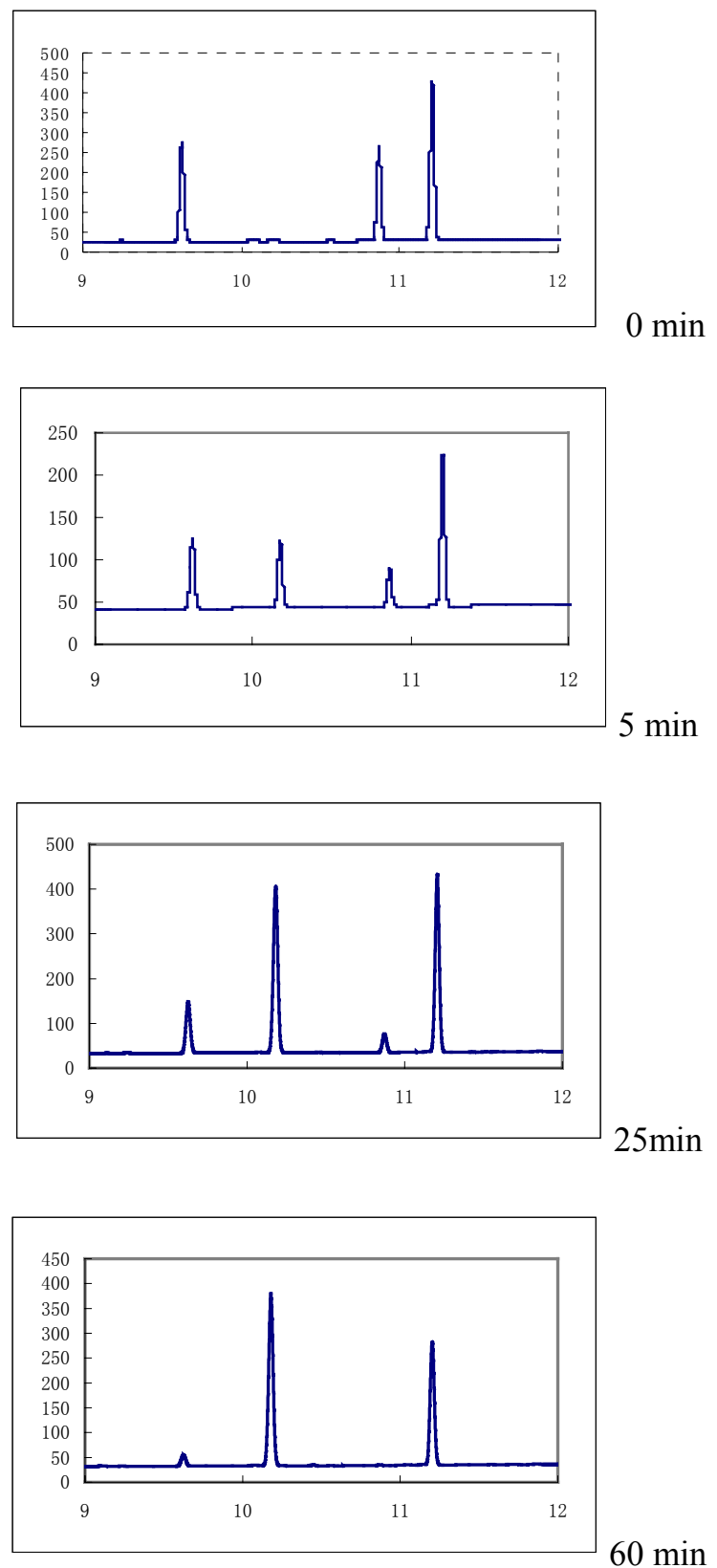

Retention Time: $\mathrm{S} 1=9.63 \mathrm{~min} ; \mathrm{P} 1=10.18 ; \mathrm{S} 11=10.87 ; \mathrm{S} 10=11.21$. 